\title{
Sección bibliográfica
}

\section{Reseñas}

Dennis A. Rondinelu. Secondary Cities in Developing Countries. Policies for Diffusing Urbanization. Sage Library of Social Research 145, Sage Publications, 1983, $288 \mathrm{pp}$.

Toda política de descentralización -y ésta parece ser prioritaria para la actual administración - debe vincularse con una política urbana nacional.

En nuestro país no existía una política expresamente urbana antes de 1978. En ese año el gobierno explicitó en el Plan Nacional de Desarrollo Urbano (PNDU) su deseo de controlar el crecimiento y distribución de la población en el territorio nacional. En él se expresaba la necesidad de ubicar equitativamente, en términos especiales, la oferta de bienes y servicios públicos para una población que se encontraba, según el diagnóstico que acompañaba al PNDU, sumamente dispersa en más de 80000 localidades de menos de 2500 habitantes o hiperconcentrada en la zona metropolitana de la ciudad de México con alrededor de 18\% de la población total del país.

Más reciente es el reconocimiento que se hace en el discurso político y en los planes de la importancia de las ciudades secundarias (intermedias y pequeñas) y su estrecha relación con los esfuerzos por desconcentrar y descentralizar la vida nacional. De ahí, por ejemplo, la atención del Consejo Nacional de Población (Conapo) a esta categoría de ciudades al organizar en nuestro país la Conferencia sobre Población y Ciudades Pequeñas y Medianas en América Latina y el Caribe los días 24 a $28 \mathrm{de}$ febrero de 1986, y la propuesta de la Secretaría de Desarrollo Urbano y Ecología (Sedue) del Programa Trianual 1986-1988 de Ciudades Medias para orientar selectivamente el gasto sectorial federal en favor de esas localidades.

Los dos temas, descentralización y ciudades secundarias, han sido preocupación de organismos internacionales como el Banco Mundial y las Naciones Unidas, y regionales como el Banco Interamericano de Desarrollo, desde fines de los años setenta. Estos temas han caracterizado además las estrategias de desarrollo en nuestro país, y parecen constituir un nuevo elemento en el esquema de desarrollo regional con el que se intenta incorporar funcional y espacialmente a toda la población que habita el territorio nacional (Graizbord, 1984).

Otros países también han introducido de una u otra manera en sus 
políticas de desarrollo urbano y regional a las ciudades secundarias. Dennis A. Rondinelli nos presenta en Secondary Cities in Developing Countries una concienzuda reseña de tales iniciativas. La sola revisión del índice invita a su lectura. En los primeros cuatro capítulos muestra argumentos contundentes en favor de estas ciudades, las define y hace referencia a su dinámica y funciones. En los últimos dos hace un recuento de las estrategias para su desarrollo y los medios administrativos y de planeación necesarios para tal fin.

Rondinelli recurre a una abundante información empírica sobre estas ciudades en todo el mundo en desarrollo. Advierte con razón -aunque para mi gusto no suficientemente- que no es del todo fácil hacer generalizaciones sobre su papel, rango-tamaño, dinámica y funciones y, por tanto, sobre las estrategias de desarrollo que necesariamente deberán responder a las caracteristicas y condiciones propias de cada pais.

Lo anterior es especialmente válido en el caso de México. De acuerdo a la información censal y a otras fuentes (Graizbord, 1986), el comportamiento de estas localidades durante la última década no concordaba plenamente con las descripciones convencionales que se han presentado para América Latina (por ejemplo, Hardoy, 1982).

En este sentido deben descartarse o tomarse con reservas algunas de las referencias sobre nuestro país que aparecen en el libro. Se entiende que el autor recurrió a información estadística muy general publicada por Naciones Unidas y que contó sólo con proyecciones para el año 1980, dado que el Censo de Población y Vivienda de 1980 no empezó a publicarse por entidad federativa sino hasta 1985 . Independientemente de este aspecto particular, el libro tiene muchas virtudes. Quizá la más importante es que puede servir de guía para el diseño y puesta en práctica de programas de acción, así como para elaborar propuestas de investigación sobre esta categoría de ciudades. Cabe señalar que el autor escoge el umbral de los 100000 habitantes para separar las ciudades medias de las pequeñas. Las primeras, dependiendo de cada país, pueden incluir localidades hasta de un millón o más habitantes, excluyendo siempre a la metrópoli nacional, generalmente la capital del país.

Pero ¿cuáles son las funciones y cuál el papel que han jugado y el que se pretende puedan jugar las ciudades medias y pequeñas?.

Cierto es que históricamente algunas de estas ciudades alcanzaron un desarrollo significativo -y así, quisiera enfatizar, seguirá sucediendo. Pero esto no se debió a un solo factor causal sino a la conjugación de varios. Los factores iniciales del crecimiento, tales como una situación y un sitio favorables caracterizados por la existencia de recursos y de ventajas estratégicas, tuvieron necesariamente que verse reforzados por otros. Uno de ellos, por demás importante en la consolidación de dichas ventajas originales y de la capacidad de adaptación a los cambios, fue la organización institucional. Otro, también muy destacado, fue la generación de eslabo- 
nes entre actividades económicas en la ciudad, lo que permitió asegurar ventajas comparativas respecto a otras localidades o regiones y atraer población e inversiones que sin duda ampliaron y consolidaron, a su vez, las relaciones interurbanas (pp. 108-109), conformando economías regionales integradas o subsistemas urbanos. Este resultado se dio en la mayoría de los países al margen de la voluntad explícita del Estado o, lo que es lo mismo, en forma no deliberada (Graizbord, 1983). Este hecho, desde mi punto de vista, queda soslayado en los argumentos presentados por Rondinelli. El autor no tiene problemas en recomendar sin reserva la intervención del Estado. Sabemos, sin embargo, que ésta es sumamente problemática principalmente en las etapas de formulación de políticas, de puesta en práctica de los planes y de evaluación de los resultados, debido en parte a la falta de mecanismos jurídicos, administrativos, fiscales, financieros, de planeación, etc., que dan por resultado inconsistencias e incompatibilidades en el conjunto de las acciones públicas.

Sintetizando múltiples referencias históricas y estudios de caso, Rondinelli señala que, si bien en general puede decirse que las ciudades secundarias en algunos países han crecido rápidamente en número y en población, y sus economías se han diversificado progresivamente, en especial en los sectores no agrícolas, queda la impresión de que realmente han jugado un papel relativamente débil en el proceso de urbanización de los países en desarrollo (pp. 177-182). En la mayoría de esos países sólo alcanzan un porcentaje pequeño de la población urbana; han crecido con lentitud si las comparamos con las metrópolis y no han sido capaces de atraer significativamente a la población migrante. En este sentido, sus posibilidades para reducir la presión demográfica de las grandes metrópolis parecerían limitadas en la mayor parte del mundo en desarrollo si las tendencias observadas continuaran. De hecho, aun cuando han podido ofrecer empleo a sus propios residentes en actividades de transformación de productos agropecuarios y en comercio y servicios, no muestran capacidad para absorber la creciente oferta de mano de obra inmigrante. Asimismo, su peso en el sector industrial manufacturero nacional ha sido menor que el de las grandes metrópolis; de ahí su incapacidad para competir con ellas. Más aún, la mayoría presenta una base económica parcialmente rural, servicios públicos y equipamiento social peores que los de las metrópolis, y una participación en actividades comerciales y de servicios proporcionalmente menor de lo que les correspondería de acuerdo a sus poblaciones.

A pesar de todo, dice Rondinelli, son muchas las funciones que las ciudades secundarias pueden desempenar y muchas las ventajas que ofrecen o se obtendrían en el plano nacional si se les incluyese en las estrategias de desarrollo zconómico y, en particular, en las políticas de desarrollo urbano y regional Debe tomarse en cuenta, como advierte Rondinelli, que entre sus funcionis se cuentan sostener empresas manufactureras y co- 
merciales pequeñas, mantener una mezcla de funciones urbanas y rurales, ofrecer servicios públicos y permitir el acceso a ellos de la población de las localidades pequeñas (rurales) de sus hinterlands (p. 177). Sin embargo, las características de su población y su estructura social, la mezcla apropiada de actividades económicas, la organización y estructura de sus economías y, especialmente, la naturaleza de la intervención pública (federal, estatal, municipal o local) son algunos de los factores que afectarán necesariamente el grado en que estas ciudades pudieran convertirse efectivamente en catalizadoras del crecimiento y desarrollo de sus regiones. Los argumentos a su favor en todo caso son innumerables y el papel que pueden desempeñar en el desarrollo urbano y regional es, de acuerdo con Rondinelli, crucial.

Estas funciones, que Rondinelli identifica con una revisión sistemática de 31 estudios de caso, son las siguientes (pp. 118-120).

1) proporcionar una localización conveniente en la descentralización de los servicios y la administración pública;

2) ofrecer economías de escala y mercados suficientemente grandes para permitir la concentración en ellas de infraestructura social (salud, educación, bienestar social y otros servicios);

3) ofrecer una amplia variedad de bienes y servicios de consumo a través del llamado "sector informal";

4) actuar como centros regionales de mercadeo en servicios de distribución, transferencia, acopio, crédito y financiamiento a la actividad productiva regional;

5) proveer de condiciones para el desarrollo de talleres artesanales y de pequeñas industrias;

6) actuar como centros de provisión para la actividad agrícola y pecuaria de las áreas rurales circundantes;

7) crear condiciones para desarrollar e incrementar la productividad y el ingreso de sus hinterlands agrícolas;

8) convertirse en una fuente de empleo e ingreso complementario para la población rural;

9) servir como centros regionales de comunicaciones y transportes;

10) bsorber migrantes rurales y convertirse en destino alternativo o escala rbana de las corrientes migratorias hacia la metrópoli nacional;

11) funcionar como centros efectivos de transformación social;

12) convertirse en centros de difusión de las innovaciones y los cambios y beneficios del desarrollo para sus regiones a través de la profusión y creación de eslabonamientos sociales, económicos y administrativos.

Dos de ellas merecen comentarios. El primero tiene que ver con el empleo en el sector informal. Las estadísticas de empleo, como señala Rondinelli (p. 34), subestiman el peso del sector terciario en los procesos sociales y económicos del desarrollo, siendo que un número sustancial de la población de bajos ingresos, de migrantes, mujeres y aun de menores 
obtienen ingresos por trabajo y ocupaciones casuales o informales. Sin embargo, el que en la mayoría de las ciudades secundarias de los países en desarrollo el sector comercio y servicios en pequeña escala y el terciario informal constituyan una fuente importante de empleo e ingresos para los residentes y la economía urbana (p. 135), no permite pensar que el fenómeno sea exclusivo de dichas ciudades. La pequeña escala de las actividades económicas tanto manufactureras como comerciales y de servicios es característica de las economías nacionales subdesarrolladas. La concentración económica y, por tanto, el número de empresas medianas y grandes o muy grandes es sumamente reducido en esos países. De tal suerte que habría que matizar la afirmación de Rondinelli y no olvidar que las medidas que se tomen de política para aprovechar, estimular o favorecer al sector informal deberán considerar que sus efectos se dejarán sentir también en actividades y población localizada en las grandes metrópolis.

El otro comentario se refiere a la importancia que parece tener en la suerte de estas ciudades la circunstancial ampliación y generación de eslabonamiento sectoriales intra e interurbanos. Cabe señalar que, en este sentido, toda política y estrategia de desarrollo urbano en favor de las ciudades intermedias y pequeñas deberá considerar dichos vínculos en el contexto de la existencia virtual o potencial de sistemas urbanos regionales (Graizbord, Negrete y Ruiz, 1983).

Al distinguir entre las localidades que cumplirían una función impulsora del desarrollo y las que, como la experiencia nos dice, han jugado un papel explotador de sus hinterlands, Rondinelli propone con base en numerosas experiencias, que para lograr lo primero deben atenderse varios factores que, creo, podrían considerarse como elementos prescriptivos (pp. 182-183). Quisiera reformularlos y presentarlos en forma condicional como preguntas. De esa manera pienso que se entendería más fácilmente la dificultad para responder al cómo hacerlo y a cuáles de las ciudades secundarias habría que estimular para que jugaran ese papel catalizador del desarrollo regional. Así:

1) ¿En qué grado las élites locales son capaces de identificar su éxito propio con el de su ciudad y región?

2) ¿En qué grado los sectores público y privado locales desean invertir recursos orientados al crecimiento y desarrollo de su ciudad y no transferir sus excedentes a otras partes? (véase, por ejemplo, Verduzco, 1984).

3) ¿Qué capacidad innovadora tienen las empresas locales para introducir recursos e innovaciones organizativas y métodos o técnicas de producción para incrementar el producto y el ingreso de la economía local?

4) ¿Hasta qué punto el gobierno federal va a apoyar realmente el crecimiento y el desarrollo de la ciudad y de su región en vez de sustraer sus recursos y transferirlos a la capital o a la economía nacional?

5) ¿En qué medida las actividades económicas de la ciudad se eslabonan en forma mutuamente benéfica con actividades de la región circun- 
dante, y qué capacidad tienen de generar efectos multiplicadores en la inversión y el empleo regionales y locales?

6) ¿En qué medida las actividades económicas en la ciudad permiten generar ingresos y, por tanto, ampliar la demanda interna de bienes y servicios producidos y distribuidos localmente? Esta prescripción, con la que no todos estarían de acuerdo, exige un breve comentario. Vale decir que, por ejemplo, la teoría de base económica señala como única fuente de ingreso para una economía regional o urbana la actividad exportadora. Por otra parte, en un libro reciente (Cities and the Wealth of Nations, Random House, 1984], Jane Jacobs argumentaba que la única estrategia de supervivencia para las ciudades pequeñas debería sustentarse en una gradual y sistemática sustitución de importaciones. Finalmente,

7) ¿Qué posibilidades existen pare fomentar una cooperación real y amplie entre el sector público y el privado de la localidad con objeto de promover actividades económicas y permitir la participación y distribución de los beneficios a las mayorías locales y, ligado a lo anterior, estimular cambios estructurales y de comportamiento social en respuesta a nuevas condiciones y necesidades que se irían presentando con el tiempo?

No hay duda de la necesidad de responder a estas preguntas. Cabe, sin embargo, preguntarse si para efectos de políticas y programas de acción (de "arriba hacia abajo") podrían identificarse categorías de ciudades y, por tanto, diseñar políticas comunes a cada clase. Quizá, si se responde afirmativamente, es válida una división del sistema urbano nacional en cuatro categorías: centros nacionales metropolitanos, centros regionales, centros urbanos y centros de servicio rural, como en principio se ha hecho en México en el PNDU, 1982 (nótese que, en todo caso, la base de clasificación es implícitamente funcional y no se refiere exclusivamente al tamaño).

El problema fundamental estriba en la selección o discriminación necesaria para lograr no sólo más eficiencia sino también mayor eficacia en las acciones que, según argumenta Rondinelli, deberán privilegiar una redistribución del ingreso si se quiere generar una mayor tasa de crecimiento económico. En otras palabras, para apoyar las inversiones directas en actividades productivas deberán hacerse inversiones de capital social que ayuden al desarrollo de los recursos humanos y su bienestar. En este sentido los elementos esenciales, según Rondinelli (p. 232), en toda estrategia de desarrollo orientada a reforzar las economías urbanas de las ciudades secundarias son cuatro:

1) aumentar la calidad y cobertura de los servicios sociales básicos en el nivel municipal;

2) mejorar la infraestructura física urbana;

3) reforzar la base económica y la estructura del empleo, y

4) crear o consolidar la capacidad administrativa, financiera y de planeación de los gobiernos locales (urbanos y municipales). 
Queda en esto último planteada la tarea de estudiar, como recomienda Richardson (cit. pp. 275-276), el proceso reciente de urbanización y las características y naturaleza del desarrollo regional, así como analizar la estructura económica, conocer los costos de producción de los servicios públicos y evaluar la capacidad para consolidar funciones urbanas de ciudades de distinto tamaño. En fin, para el conjunto de ciudades, reflexionar sobre cuál debería ser el compromiso entre un sistema jerárquico necesario para la producción y distribución territorial de bienes y servicios y aquél que se requeriría para la transmisión y difusión de impulsos de crecimiento e innovaciones en el territorio nacional.

Rondinelli nos ofrece en este libro elementos para eso y más. Su aportación al estudio de las ciudades intermedias y pequeñas y, por ende, al diseño de políticas de desarrollo urbano hace imprescindible su lectura por parte de académicos, planificadores y, en general, tomadores de decisiones y diseñadores de tales políticas.

Boris Graizbord

El Colegio de México

\section{Bibliografía}

Graizbord, Boris (1983), “Comment”, en Regional Development Dialogue, vol. 4, núm. 2, 42-44.

(1984), "Desarrollo regional, ciudades intermedias y descentralización en México: observaciones críticas al Plan Nacional de Desarrollo Urbano (1978-1982)', en Demografía y Economía xviII, 1, núm 57, 27-47.

(1986), "Crecimiento urbano en México: cambios recientes", ponencia presentada en el II Congreso Iberoamericano de Urbanismo, Tlaxcala, México, 21-25 de abril de 1986.

Graizbord, Boris, M.E. Negrete y C. Ruiz (1983), “Desarrollo urbano, sistema de ciudades y descentralización en México: propuesta de análisis”, ponencia presentada en la Segunda Reunión Subregional BID-Colmex sobre descentralización y desarrollo urbano. México, 17-21 de enero de 1983, mimeo.

Hardoy, J. (1982), "Urban Development and Planning in Latin America", en Regional Development Dialogue, vol. 1, núm. 3, 1-23.

Verduzco, Gustavo (1984), "Crecimiento urbano y desarrollo regional: el caso de Zamora, Michoacán”, en Revista Interamericana de Planificación, vol. xvrI, núm 71 .

Emilo Pradilla. Contribución a la crítica de la "teoría urbana". Universidad Autónoma Metropolitana- Xochimilco, México, 1984, $731 \mathrm{pp}$.

En los últimos tres lustros la sociclogía urbana latinoamericana viene desarrollándose bajo el influjo de las teorizaciones efectuadas por investiga- 
dores que se reclaman marxistas, tales como Castells, Lojkine, Topalov, Borja y otros. Su producción está ampliamente difundida y, mal que bien, orienta muchos de los libros, tesis y artículos que surgen en la temática.

Lo anterior marca la importancia del libro de Emilio Pradilla que, inserto en el "paradigma", pretende discutir los conceptos y teorías elaborados por los autores citados. Emilio Pradilla no requiere mayor presentación. Sus aportes, que tienden a ubicarse en el campo de la producción teórica, son ampliamente conocidos, en especial sus análisis de los procesos de "autoconstrucción" (Arquitectura y autogobierno, núm 7, UNAM, 1977).

Esta breve reseña contrasta con un libro tan amplio, por lo que nos limitaremos a señalar su posición en torno a aquellas conceptualizaciones que pensamos son de mayor uso en América Latina: la teoría particular de lo urbano, los llamados medios de consumo colectivos y el carácter de las contradicciones urbanas.

En La cuestión urbana (1972) Manuel Castells había planteado la necesidad marxista de producir una teoría que diera cuenta de los elementos de la estructura espacial, distinguiendo los sistemas económico, político e ideológico. Su punto de partida era el concepto de espacio, visto como producto material bajo determinadas relaciones sociales que le otorgan una función social. De lo que se trataba, entonces, era de establecer las leyes estructurales y coyunturales que rigen su existencia y transformación.

Pradilla cuestiona el concepto de espacio como punto de partida, por considerar que el propio concepto - y no su teorización específica- posee un carácter ideológico. En ese sentido, el concepto de espacio sería definido, sin significado propio y, además, una piedra angular de teorizaciones no marxistas a las que el propio Castells ha críticado acertadamente. En cuanto al desarrollo del discurso de Castells, Pradilla considera que trabaja con un criterio reduccionista que, por lo tanto, no es fiel al desarrollo del propio Marx. Así, por ejemplo, considera que Castells hace coincidir producción con proceso de trabajo. Como consecuencia de esa reducción Castells subvalora el papel de la producción (léase actividad industrial) enfatizando el momento del consumo, lo que constituirá uno de los pilares de su teorización.

Acto seguido Pradilla sostendrá alternativamente dos afirmaciones: en primer lugar, descarta el concepto de espacio y opta por la relación naturaleza-sociedad como proceso de "apropiación transformación-destrucción"; consecuentemente, en segundo lugar, afirmará que no son necesarias teorías "particulares" o "específicas" para estudiar los fenómenos urbanos, sino que ellos pueden ser aprehendidos por los conceptos y categorías del "materialismo histórico-dialéctico", a condición de construir conceptos descriptivos específicos. En sus palabras, "no se pretende construir conceptos teóricos, sino descriptivos, instrumentales, intermedios y generales que sirvan para la explicación de los conceptos" (p. 85). 
En esa línea plantea utilizar dos conceptos descriptivos. El primero es el de soporte material de la vida social, entendido como el conjunto de "objetos materiales resultantes de un proceso voluntario y consciente de transformación de la naturaleza . . . para satisfacer cualquiera de las necesidades sociales históricamente determinadas"'; el segundo es el de soportes materiales de las formaciones sociales capitalistas, por el que entiende los soportes que se generan por la combinación desigual de diferentes modos de producción en una sociedad concreta. Tales conceptos descriptivos reemplazarían aquello que se denomina "espacio".

La crítica al concepto de medios de consumo colectivo (MCC) es la crítica a la base del edificio teórico levantando por Lojkine, Castells y otros. En efecto, para esas teorizaciones, tal concepto es el elemento básico de la estructura urbana, el "objeto" de las políticas urbanas y el nudo de la contradicción estructural sobre el que se levantan los movimientos urbanos. Recordemos que ese concepto fue utilizado para dar cuenta de fenómenos que Marx no conoció y que amplía el concepto marxista de condiciones generales de producción. En los MCC se consideran los servicios de energía, agua, transporte, educación, salud e, incluso la vivienda.

El cuestionamiento de Pradilla apunta, en este caso, a denunciar su carácter general y abstracto, que lo inutiliza para el análisis concreto. En otras palabras, la generalidad de los MCC no permite distinguir los diferentes fenómenos de que el concepto intenta dar cuenta. Así, alternativamente, plantea conservar el concepto de condiciones generales de la producción para aquella parte que se articula al proceso inmediato de producción y de circulación de mercancías (energía, agua potable, transporte, etc). Propone, por otra parte, el concepto de condiciones generales de reproducción de los trabajadores y los no trabajadores, buscando incluir las condiciones de reproducción de la burguesía y no sólo, como propone Lojkine, de la fuerza de trabajo. En esta medida, Pradilla critica las propuestas que identifican la ciudad como "espacio de reproducción de la fuerza de trabajo" exclusivamente.

Asimismo, Pradilla critica las teorizaciones que mantienen que los MCC sirven sólo a los trabajadores, olvidando a los sectores dominantes; igualmente critica que se sostenga que los MCC no son mercancías, cuando su análisis muestra que sí lo son. Este error obedecería al descuido del momento de la producción de los llamados MCC (energía eléctrica, agua potable, etc) y, en este sentido, niega enfáticamente que la vivienda no sea una mercancía cuando es producida por el Estado.

Como corolario del énfasis de Pradilla en la producción (y no en el consumo) se arriba al nudo gordiano en la comprensión de los movimientos urbanos: ¿se localizan sus contradicciones en la esfera del consumo o de la producción? La respuesta de Pradilla es enfática: las contradicciones sociales no ocurren en el consumo sino en la producción, recordando los análisis de Marx de que la producción determina el consumo. 
En esta línea, al rescatar el momento de la producción de las condiciones generales de reproducción de la población (energía, transporte, salud, educación, etc) y su carácter de mercancías, Pradilla señala que es en las relaciones burguesas de distribución del producto social en las que hay que encontrar la explicación de las contradicciones urbanas.

Esta consideración es importante en la medida que autores como Castells ubican las contradicciones urbanas en la esfera del consumo, autonomizándola relativamente en la esfera de la producción. Será esta autonomía entre ambas esferas la que le permitirá sostener, por ejemplo, la alianza en los movimientos ciudadanos de clases sociales enfrentadas en la producción. Esta supuesta unidad en el consumo de adversarios en la producción será criticada por Pradilla.

Ciertamente que los movimientos urbanos no están específicamente tratados en el libro. La crítica a las conceptualizaciones de Castells, Borja y otros autores sobre este tema será materia de una segunda obra, de la cual conocemos un primer borrador elaborado en 1981 con el título de La cuestión urbana y la lucha de clases.

Para finalizar quisiéramos señalar que la principal virtud del libro es la manera rigurosa en que plantea propuestas teóricas al interior del "paradigma" marxista. Se trata de propuestas que deben ser sometidas a la discusión por los investigadores urbanos y que quizás permitan replantear algunas líneas de análisis.

Julio Calderón

DESCO - Lima

\section{Notas}

MARIO MARgUlis y RODOlFo TUIRÁN. "Desarrollo y población en la frontera norte, el caso de Reynosa”. El Colegio de México, México, 1986, $323 \mathrm{pp}$.

En este libro los autores emprenden la investigación de la evolución económica y demográfica de la ciudad de Reynosa, como caso particular de la frontera norte del país.

El estudio se basa principalmente en una encuesta levantada en Reynosa en 1980 y en los censos generales de población y vivienda de 1980.

Los resultados de la investigación se presentan en ocho capítulos que se dedican a: introducción de los conceptos principales y formulación de hipótesis generales; evolución histórica de Reynosa; descripción y z nálisis de las tendencias poblacionales registradas en Reynosa entre 1! $30 \mathrm{y}$ 
1980; presentación de indicadores socioeconómícos globales que ubican en el contexto nacional y regional a la ciudad de Reynosa; descripción y análisis de las características de la inmigración; niveles y formas de participación de la población en la actividad económica y las características del mercado de trabajo; la ocupación y la reproducción social desde el punto de vista de las unidades domésticas.

Las principales conclusiones y hallazgos que se derivan de la investigación son: las modalidades del crecimiento poblacional del decenio 1970-1980 difieren notablemente de las observadas en periodos anteriores; el crecimiento social en las principales ciudades fronterizas se ha reducido a niveles mínimos o a tasas negativas durante 1970-1980; en los principales municipios fronterizos se observan, en el mismo periodo, tasas negativas de crecimiento social. En las zonas rurales de esos municipios de la frontera las tesas negativas de crecimiento social son muy elevades y se remontan al decenio 1960-1970; la eusencia casi total de crecimiento social no implica la desaparición de la inmigración a las ciudades fronterizas. Se encontró que ha existido también durante la última decada una emigración importante que compensó y en ocasiones desbordó los aportes inmigratorios.

M.E.M.C.

HeNRI LABORIT. L'homme et la ville. Flammarion, París, 1971, 214

pp.

Este libro representa el resultado de la búsqueda de un grupo de estudiantes que, dirigidos por el autor, realizaron en forma experimental un enfoque biológico del fenómeno urbano; su propósito es proporcionar elementos de reflexión que constituyan una base para futuras investigaciones.

Se adopta en esta obra un enfoque dinámico global que busca explicar el fenómeno urbano y su vinculación con el hombre y su entorno; para ello, se utilizan los principios de la cibernética como instrumento de análisis.

La investigación no trata de establecer analogías simplistas entre estructura urbana y estructura biológica. En realidad, parte de la afirmación de que la finalidad de los organismos vivos, cualquiera que sea su nivel de organización, es mantener su estructura. En este orden de ideas, la finalidad del grupo humano, como organismo social, es mantener su estructura compleja. La construcción de una ciudad no es la finalidad del grupo humano; no es más que un medio indirecto que sirve al grupo humano para mantener su estructura.

Para entender el fenómeno urbano es preciso profundizar en el cono- 
cimiento de las motivaciones subyacentes del grupo humano, en última instancia de las leyes que gobiernan la conducta humana en sociedad, mismas que preceden al establecimiento de las estructuras sociales y dan nacimiento a la ciudad, a la organización del espacio que las rodea.

El autor sostiene que el impulso primitivo de dominación es el origen de la estructura y formación de las clases sociales y que el hombre ha utilizado la ciudad, desde sus inicios, como un espacio donde todo está arreglado para lograr la dominación de unos individuos sobre otros; la ciudad funciona para asegurar la defensa de la propiedad de los objetos, los individuos, los medios de producción y aun los niveles jerárquicos.

En la ciudad moderna, la ganancia y la acumulación del capital, la producción de mercancías, la expansión económica inexorable no son más que los medios utilizados por los individuos más agresivos para establecer y mantener su dominación sobre la masa.

Actualmente, en la civilización industrial, también resultado del instinto de dominación, se presenta el llamado de atención de los ecologistas: o el hombre cambia su comportamiento o está condenado a desaparecer como especie.

M.E.M.C

DANIEL HIERNAUX. "Urbanización y autoconstrucción de vivienda en Tijuana”'. Centro de Ecodesarrollo, México, 1986, 146 pp.

La investigación que se presenta en este libro forma parte de un programa que tiene como objetivo estudiar los procesos de urbanización y de construcción de la vivienda popular en ciudades claves del país.

El trabajo se divide en dos partes. La primera está conformada por cuatro capítulos, que analizan: la formación del espacio fronterizo, con especial énfasis en la idea de que en dicho espacio se manifiestan las contradicciones y desigualdades en las relaciones México-Estados Unidos; el contexto económico de la frontera norte y en particular el de Tijuana y, finalmente, el proceso urbano de Tijuana.

La segunda parte se enfoca al tema de la autoconstrucción de la vivienda, apoyándose en el análisis de los resultados de una encuesta levantada en 1982. Esta parte la conforman cinco capítulos que desarrollan los siguientes temas: el perfil del encuestado; el perfil demográfico del autoconstructor y su inserción en el mercado de trabajo; la inserción urbana del autoconstructor y su movilidad urbana; el proceso de producción de la vivienda, el uso de materiales y la aplicación de la fuerza de trabajo y de capital en la construcción de la vivienda; y en el quinto rubro se presentan las conclusiones y propuestas.

Entre las conclusiones que se derivan de la investigación cabe men- 
cionar las siguientes. Tijuana es una ciudad con un alto crecimiento demográfico social; su particularidad -como la de las demás ciudades fronterizas-es que los migrantes no la eligen como lugar de residencia, sino como lugar de paso. Esta circunstancia genera zonas de transición para migrantes con la presencia de vivienda transitoria de ocupación efímera y la presión sobre el mercado inmobiliario. La influencia de la vecindad con Estados Unidos se hace sentir en los usos y la disponibilidad del suelo y genera la existencia de grandes especuladores públicos y privados.

La autoconstrucción - que el autor prefiere llamar autoproducción, porque en ese término se incluyen todo tipo de acciones además de la de construcción--, se abastece de materiales de desecho de los Estados Unidos, en parte por la preferencia al uso de productos norteamericanos, pero en parte, también, debido a la escasez del abasto de materiales nacionales.

El entorno en donde se realiza la autoconstrucción se caracteriza por la precariedad de las condiciones de vida: el equipamiento social y urbano es casi inexistente; el transporte funciona mal y los asentamientos son ilegales.

No se da la presencia del Estado en el proceso de autoconstrucción, pero en cambio las normas y planes que éste genera obstaculizan o reducen las posibilidades de que el individuo de bajos ingresos tenga acceso a una vivienda.

Hiernaux propone que el Estado continúe absteniéndose de intervenir en el proceso de autoconstrucción y sugiere que sean los diversos grupos de la sociedad civil, con la participación directa del ocupante, quienes tomen el control del proceso.

M.E.M.C

David HaRvey. The Urbanization of Capital. Studies in the History and Theory of Capitalist Urbanization. The Johns Hopkins University Press, 1985, Baltimore, $239 \mathrm{pp}$.

El principal objetivo del autor es entender el proceso de urbanización en el capitalismo; para lograrlo, considera que las categorías marxistas son la únicas que permiten su estudio riguroso y científico. Está consciente, sin embargo, de que buscar una explicación de la urbanización en términos marxistas es acudir a un marco teórico controvertido y, en algunos aspectos, incompleto. El autor aclara que no concibe la urbanización como objeto específico de análisis separado de lo que es el capitalismo; por el contrario, considera que el estudio de la urbanización es el estudio del 
proceso del capital. Asimismo, declara que se reduce a la investigación de las formas capitalistas de urbanización porque sostiene la idea de que el término "urbano" tiene un significado específico en el modo de producción capitalista, que no puede aplicarse a otros contextos sociales sin alterar radicalmente su significado.

En esa línea de pensamiento, en los ocho capítulos que conforman el libro, el autor procede al análisis de diversos temas, como el proceso de urbanización en el capitalismo; la geografía de la acumulación capitalista; la renta de la tierra y la renta monopólica; la teoría de la diferenciación residencial; la ideología de la planificación y la urbanización del capital.

Resulta de interés destacar que en el último capítulo Harvey comenta que diez años atrás se había preguntado si se podría derivar una explicación teórica e histórica del proceso urbano en el capitalismo a partir del estudio-de las supuestas leyes del movimiento del modo de producción capitalista. Después de una década de reflexión sobre el tema llega a la conclusión de que es necesaria una reformulación de la pregunta en los siguientes términos: ¿cómo se urbaniza el capital? y ¿cuáles son las consecuencias de esa urbanización?

Harvey considera que la respuesta tiene implicaciones profundas para entender el futuro del capitalismo, así como sobre las perspectivas de transición a algún modo de producción alternativo. En el capítulo final explica la forma en que el capitalismo crea y recrea espacios físicos y sociales, y cuáles son las contradicciones que surgen en ese proceso de urbanización, a través de un recorrido histórico que se inicia en el mercantilismo hasta llegar a la ciudad postkeynesiana.

M.E.M.C.

David HaRvey. Consciousness and the Urban Experience Studies in the History and Theory of Capitalist Urbanization. The Johns Hopkins University Press, Baltimore, 1985, 293 pp.

Aunque esta obra puede leerse en forma independiente, su publicación es paralela a la de The Urbanization of Capital en la que el autor presenta su teoría respecto del proceso de urbanización, al que ubica dentro de los procesos generales de producción y acumulación capitalista.

En este libro el autor considera que el análisis del proceso urbano permite encontrar las raíces de la formación de la conciencia de los individuos acerca de las realidades materiales de la vida diaria. De ahí se extraen los significados de espacio y tiempo, de poder social y su legitimación, de las formas de dominación e interacción social, y de la naturaleza humana, sociedad civil y vida política. 
Harvey afirma que la urbanización de la conciencia tiene que tomarse como un fenómeno real: social, cultural y político. Este tema es muy dif́́cil de fundamentar teóricamente, dado el aparato teórico disponible. Por ello -explica el autor- los estudios sobre la conciencia y la experiencia urbana son especulativos y se sustentan principalmente en la experiencia histórico-geográfica.

En el primer capítulo, Harvey pretende entender las fuerzas que enmarcan el proceso urbano y la experiencia urbana en el capitalismo. Argumenta que la existencia del dinero como intermediario en el intercambio de mercancías transforma y determina los significados de tiempo y espacio en la vida social, y define límites e impone necesidades a la urbanización.

Dentro de este argumento general pretende construir otro que ayude a entender el significado político de la protesta urbana, las formas del poder local y las diferentes experiencias urbanas.

En el siguiente capítulo, el autor busca establecer un marco teórico para entender la lucha de clases en el capitalismo avanzado. Su argumento central es que la sociedad capitalista necesita crear una base material para los propósitos de la producción y reproducción; este proceso está lleno de contradicciones y tensiones y las relaciones de clase de la sociedad capitalista inevitablemente engendran conflictos.

Los conceptos teóricos se ilustran en los capítulos 3 y 4 con una descripción detallada de los cambios que se presentaron en París, en el siglo pasado (incluyendo la Revolución de 1848 y la experiencia de la Comuna de París) y con un análisis referido a la construcción de la Basílica del Sagrado Corazón.

En el capítulo 5, el autor presenta una explicación de la creación y control del espacio y clasifica el proceso urbano como "geografía histórica de la lucha de clases y de la acumulación del capital". Al examinar los detalles del proceso de creación física y social, demuestra cómo el capitalismo define la ciudad y la conciencia de sus habitantes y al mismo tiempo señala que el proceso de urbanización capitalista puede obstaculizar e impedir la evolución futura del capitalismo. 


\section{B. CROUSSE, E. LE BRIS Y E. LE ROY (compiladores y presentadores). Espaces disputés en Afrique Noire. (Practiques foncières locales). Karthala Ministerio Belga de la Educación Nacional ORSTOM CNRS, París, 1986, 422 pp.}

En este volumen se publica una parte de las ponencias presentadas en el coloquio internacional realizado en Francia, a fines de 1983, sobre el tema "Políticas locales con respecto al suelo, para la producción y reproducción del espacio en África Negra".

El libro consta de una introducción general, seis capítulos y una parte final de conclusiones. La introducción presenta algunos antecedentes del coloquio y aclara ciertos elementos conceptuales, entre los cuales destaca el de "matriz espacio-temporal", aplicado básicamente a dos formas de representar el espacio (la matriz autóctona y la matriz capitalista). Asimismo se afirma que las prácticas actuales referidas al suelo se aplican a dos esferas de la organización social: las políticas del territorio y el control de la fuerza de trabajo.

En los seis capítulos mencionados se tratan, básicamente a través de estudios de caso, los siguientes temas: las prácticas del Estado y del capital con respecto al suelo en algunas metrópolis; los problemas de los espacios industriales en los que el Estado está implicado en diferentes grados; las prácticas locales en centros urbanos secundarios; las agroindustrias y los grandes conglomerados; los obstáculos impuestos por el suelo y las actividades productivas en el medio campesino.

En las conclusiones se pone énfasis en la problemática pluridisciplinaria planteada por la red que organizó el coloquio, potencialmente generadora de resultados novedosos a partir de estudios particulares, los que lejos de oponerse, son susceptibles de generar progresivamente una visión globalizante de la relación espacio-problemática del suelo.

Si bien el tipo de análisis propuesto por los organizadores del evento forzó a veces a los autores de los estudios de caso a introducir en el analisis al Estado, los coordinadores de Espaces disputés llegan a la conclusión que este constituye un actor fundamental dentro de las prácticas relativas al suelo, en África. 